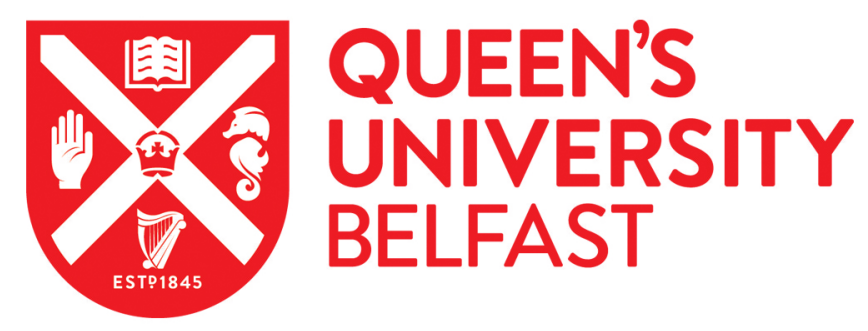

\title{
Dynamic control of laser driven proton beams by exploiting self- generated, ultrashort electromagnetic pulses
}

Kar, S., Ahmed, H., Nersisyan, G., Brauckmann, S., Hanton, F., Giesecke, A. L., Naughton, K., Willi, O., Lewis, C. L. S., \& Borghesi, M. (2016). Dynamic control of laser driven proton beams by exploiting self-generated, ultrashort electromagnetic pulses. Physics of Plasmas, 23(5), [055711]. https://doi.org/10.1063/1.4948725

Published in:

Physics of Plasmas

Document Version:

Publisher's PDF, also known as Version of record

Queen's University Belfast - Research Portal:

Link to publication record in Queen's University Belfast Research Portal

\section{Publisher rights}

Copyright 2016 AIP Publishing

This article may be downloaded for personal use only. Any other use requires prior permission of the author and AIP Publishing

The following article appeared in Kar, S, Ahmed, H, Nersisyan, G, Brauckmann, S, Hanton, F, Giesecke, AL, Naughton, K, Willi, O, Lewis,

CLS \& Borghesi, M 2016, 'Dynamic control of laser driven proton beams by exploiting self-generated, ultrashort electromagnetic pulses'

Physics of Plasmas, vol 23, no. 5 and may be found at http://scitation.aip.org/content/aip/journal/pop/23/5/10.1063/1.4948725

\section{General rights}

Copyright for the publications made accessible via the Queen's University Belfast Research Portal is retained by the author(s) and / or other copyright owners and it is a condition of accessing these publications that users recognise and abide by the legal requirements associated with these rights.

Take down policy

The Research Portal is Queen's institutional repository that provides access to Queen's research output. Every effort has been made to ensure that content in the Research Portal does not infringe any person's rights, or applicable UK laws. If you discover content in the

Research Portal that you believe breaches copyright or violates any law, please contact openaccess@qub.ac.uk. 


\section{AIP $\left.\right|_{\text {Physics of }}$

Dynamic control of laser driven proton beams by exploiting self-generated, ultrashort electromagnetic pulses

S. Kar, H. Ahmed, G. Nersisyan, S. Brauckmann, F. Hanton, A. L. Giesecke, K. Naughton, O. Willi, C. L. S. Lewis, and M. Borghesi

Citation: Physics of Plasmas 23, 055711 (2016); doi: 10.1063/1.4948725

View online: http://dx.doi.org/10.1063/1.4948725

View Table of Contents: http://scitation.aip.org/content/aip/journal/pop/23/5?ver=pdfcov

Published by the AIP Publishing

\section{Articles you may be interested in}

Ultrafast proton radiography of the magnetic fields generated by a laser-driven coil current

Phys. Plasmas 23, 043106 (2016); 10.1063/1.4945643

Generation and pointing stabilization of multi-GeV electron beams from a laser plasma accelerator driven in a pre-formed plasma waveguidea)

Phys. Plasmas 22, 056703 (2015); 10.1063/1.4919278

Bidimensional Particle-In-Cell simulations for laser-driven proton acceleration using ultra-short, ultra-high contrast laser

Phys. Plasmas 21, 123104 (2014); 10.1063/1.4902125

Measurements of energy and angular distribution of hot electrons and protons emitted from a $\mathrm{p}$ - and $\mathrm{s}$ polarized intense femtosecond laser pulse driven thin foil target

Phys. Plasmas 13, 043104 (2006); 10.1063/1.2192758

Dependence on laser intensity and pulse duration in proton acceleration by irradiation of ultrashort laser pulses on a $\mathrm{Cu}$ foil target

Phys. Plasmas 12, 073102 (2005); 10.1063/1.1943436

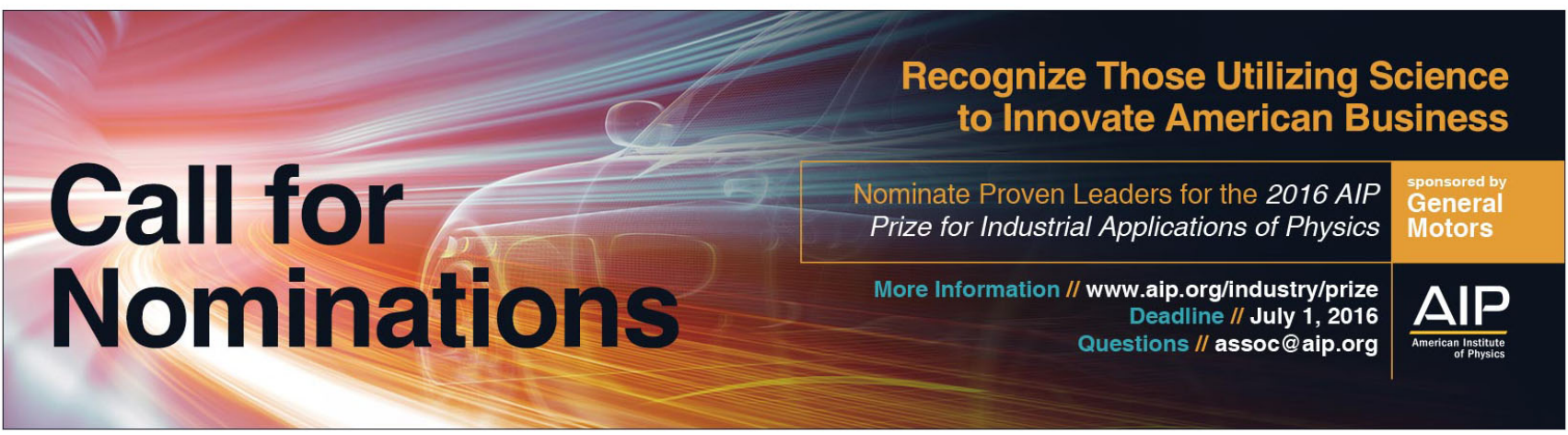




\title{
Dynamic control of laser driven proton beams by exploiting self-generated, ultrashort electromagnetic pulses
}

\author{
S. Kar, ${ }^{1, a), b)}$ H. Ahmed, ${ }^{1}$ G. Nersisyan, ${ }^{1}$ S. Brauckmann, ${ }^{2}$ F. Hanton, ${ }^{1}$ A. L. Giesecke,${ }^{2}$ \\ K. Naughton, ${ }^{1}$ O. Willi, ${ }^{2}$ C. L. S. Lewis, ${ }^{1}$ and M. Borghesi ${ }^{1}$ \\ ${ }^{1}$ Centre for Plasma Physics, School of Mathematics and Physics, Queen's University Belfast, Belfast BT7 INN, \\ United Kindom \\ ${ }^{2}$ Institut für Laser-und Plasmaphysik, Heinrich-Heine-Universität, Düsseldorf, Germany
}

(Received 1 January 2016; accepted 25 April 2016; published online 27 May 2016)

As part of the ultrafast charge dynamics initiated by high intensity laser irradiations of solid targets, high amplitude EM pulses propagate away from the interaction point and are transported along any stalks and wires attached to the target. The propagation of these high amplitude pulses along a thin wire connected to a laser irradiated target was diagnosed via the proton radiography technique, measuring a pulse duration of $\sim 20 \mathrm{ps}$ and a pulse velocity close to the speed of light. The strong electric field associated with the EM pulse can be exploited for controlling dynamically the proton beams produced from a laser-driven source. Chromatic divergence control of broadband laser driven protons (upto $75 \%$ reduction in divergence of $>5 \mathrm{MeV}$ protons) was obtained by winding the supporting wire around the proton beam axis to create a helical coil structure. In addition to providing focussing and energy selection, the technique has the potential to post-accelerate the transiting protons by the longitudinal component of the curved electric field lines produced by the helical coil lens. Published by AIP Publishing. [http://dx.doi.org/10.1063/1.4948725]

\section{INTRODUCTION}

Since its first experimental observation, target normal sheath acceleration (TNSA) has been the most studied ion acceleration mechanism using high intensity, short pulse lasers. ${ }^{1}$ While the brightness, laminarity, and pulse duration of the TNSA beams are uniquely attractive and markedly different from those of conventional accelerator beams, there are some inherent shortcomings associated with the ion beams produced through this mechanism. For instance, their inherent divergence (typically $40^{\circ}-60^{\circ}$, depending on the laser and target parameters ${ }^{2}$ ) makes it difficult to utilize the full proton flux for applications, or for further transport and beam manipulation. In order to maintain a suitably high ion flux over the distances required for applications such as radiobiology, ${ }^{3}$ warm dense matter creation, ${ }^{4}$ and neutron source development, ${ }^{5,19}$ some means of constraining the beam divergence are needed. The manipulation of laser generated proton beams presents specific challenges due to the high bunch charge and short pulse nature of the beams. Additional constraints of the TNSA mechanism are the broad, exponential energy spectrum and slow $\left(\propto I^{1 / 2}\right)$ energy scaling with laser intensity $(I)$. While some of the focussing techniques, such as laser-driven micro-lenses, ${ }^{6}$ magnetic quadrupoles, ${ }^{7}$ pulsed solenoids, ${ }^{8}$ allow a degree of spectral tailoring in addition to the divergence control, they typically capture a small portion of the divergent proton beam. Other techniques such as ballistic focussing from curved targets ${ }^{9}$ or self-driven quasi-electrostatic lenses ${ }^{10}$ act on the full beam, but they either provide

\footnotetext{
Note: Paper YI2 5, Bull. Am. Phys. Soc. 60, 391 (2015).

${ }^{a)}$ Electronic mail: s.kar@qub.ac.uk

${ }^{\mathrm{b})}$ Invited speaker.
}

achromatic focussing or only partial reduction in divergence. Therefore, innovative approaches are required to enable beam control and optimisation.

Here, we discuss a recently developed scheme to achieve focusing, energy selection, and post-acceleration of the proton beams simultaneously, ${ }^{18}$ by utilising a high amplitude electromagnetic (EM) pulse, self-generated following the interaction of intense lasers with solid targets. The EM pulse effectively carries a neutralizing current which attempts to restore charge neutrality in the irradiated region of the target, following its positive charge-up due to the prompt escape of a fraction of hot electrons produced during the interaction. ${ }^{10-12}$ The experimental data characterising the propagation of the pulse are presented, followed by data showing chromatic focussing of the TNSA proton beam. The EM pulse propagation was observed by employing a self proton probing (SPP) technique ${ }^{13}$ which employs the same principles of the standard proton radiography technique, ${ }^{12,14,20}$ but in an arrangement requiring only one laser beam. The high temporal and spatial resolution of the radiography technique ${ }^{14}$ enabled mapping the ultrafast flow of the pulse along the wire, and observing that it retains its pulse shape over several centimetres of propagation. The extremely strong $(\sim \mathrm{GV} / \mathrm{m})$, localised electric field associated with the EM pulse can be exploited to manipulate the beam parameters of the TNSA-driven MeV protons. Allowing the pulse to travel along a helical path around the protons beam (by attaching a helical coil made of a thin wire to the rear side of the proton generating foil), the potential of manipulating the proton beam parameters was studied. By varying the physical dimensions of the helical coil, experimental data demonstrate highly effective, and intrinsically chromatic proton beam guiding. 


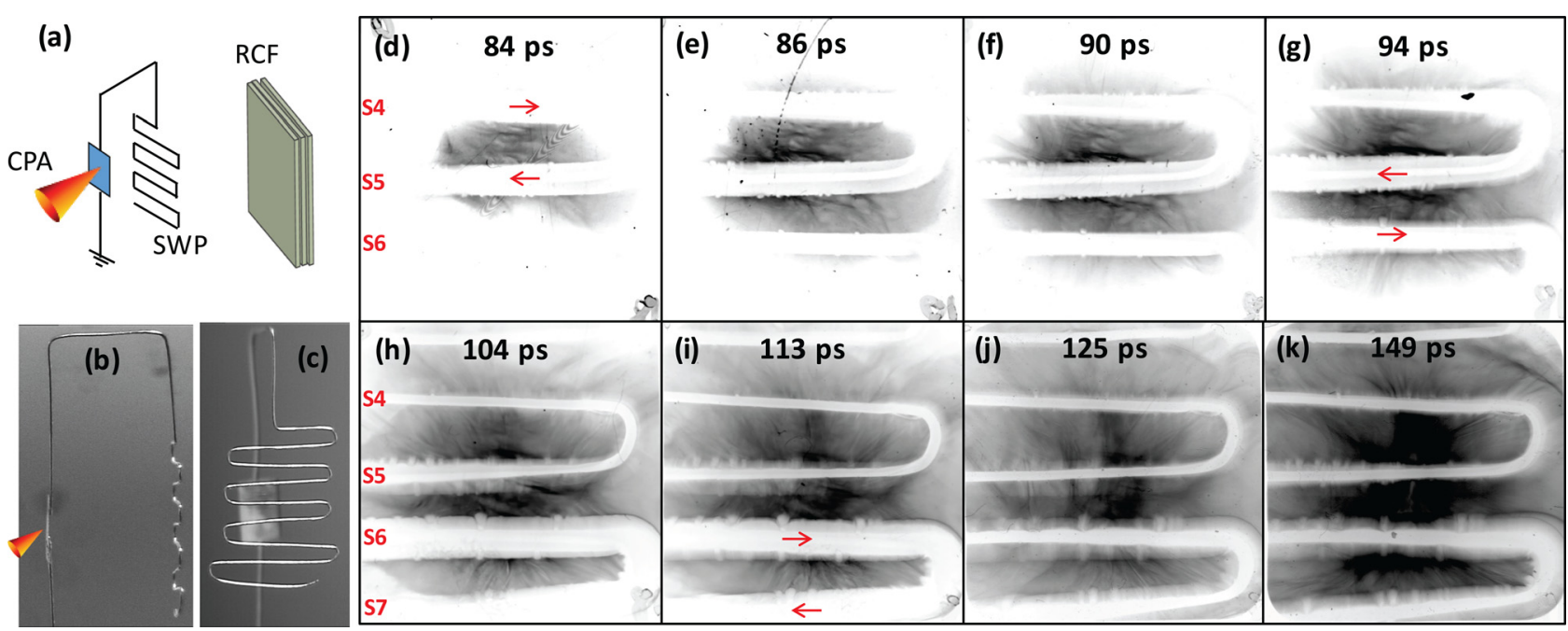

FIG. 1. (a) Schematic of the experimental setup used for probing the EM pulse propagation in a wire connected to a laser irradiated target in a self proton probing arrangement. (b) and (c) Side and rear views of the target used in the experiment, taken with a 8 bit CCD camera. The length of each horizontal wire segment in the SWP was $\sim 3 \mathrm{~mm}$, the vertical spacing between two segments was $\sim 750 \mu \mathrm{m}$, and there were 9 segments in the SWP. The length of the Al wire from the Au foil to top of the winding in the SWP was chosen $(\sim 11.5 \mathrm{~mm})$, such that a pulse travelling at the speed of light would be intercepted by the probe proton beam. The distance between the proton source and the centre of the SWP was $\sim 3.7 \mathrm{~mm}$, whereas the RCF stack detector was placed at $\sim 26 \mathrm{~mm}$ from the proton generating foil, providing a magnification of $\sim 7$ in the point-projection arrangement. (d)-(k) Raw proton images of the charge flow along the SWP at different probing times (as labelled at the top of each image), obtained in a single shot from different RCF layers in the stack detector. The horizontal wire segments are labelled as S3, S4, etc., in the proton images, and the arrows indicate the direction of the current flow through the SWP from top to bottom.

\section{EXPERIMENTAL SETUP}

The data were collected during an experiment carried out using the TARANIS laser at Queen's University Belfast. ${ }^{15}$ The laser operates with the chirped pulse amplification technique delivering pulses of $\sim 600$ fs duration with energy $\sim 5 \mathrm{~J}$ on target. The laser was focused by a $\mathrm{f} / 3$ off axis parabola onto $\sim 10 \mu \mathrm{m}$ thick and a few $\mathrm{mm}^{2}$ wide gold foil at an intensity $\sim 2 \times 10^{19} \mathrm{~W} \mathrm{~cm}^{-2}$. In this interaction, protons are accelerated from the rear surface of the foil via the TNSA process. A stack of multilayer radiochromic films (RCFs) of type $\mathrm{HD} 810^{16}$ was used as a proton detector. Due to the Bragg peak energy deposition profile of protons in matter, the proton dose deposited in a given layer of RCF is due primarily to a narrow range of proton energy, defined by the position of the RCF layer in the stack.

For the first part of the experiment, the proton beam was used as a charged particle probe in a self-proton probing (SPP) arrangement ${ }^{13}$ i.e., the protons are used to probe a portion of the target from which they are generated. During the laser interaction, the target becomes positively charged due to the immediate escape of hot electrons. As part of the ultrafast charge dynamics induced by the intense laser irradiation of the target, an EM pulse is launched from the interaction region which travels along any stalks/wires attached to the target. Therefore, a special target design was used, as shown in Fig. 1(a), in order to follow the propagation of the pulse along a wire connected to the proton generating foil. The field of view of the probe protons at a few $\mathrm{mm}$ from the proton source is a few $\mathrm{mm}$ wide due to the intrinsic divergence $^{2}$ of the proton probe. Therefore, in order to maximise the length of the wire observed within the field of the view of the proton probe, the $\mathrm{Al}$ wire was folded to a square wave pattern (SWP) in front of and parallel to the interaction foil as shown in Fig. 1(a).
The technique of exploiting the ultra-fast EM pulse towards control and optimisation of the proton beam parameters is based on deploying a coil structure attached to the rear side of the proton generating coils, as shown in Fig. 2(a). Helical coils of cylindrical geometry of different dimensions were used to observe the effect of the travelling pulse along the coil windings on the transiting broadband, divergent beam of protons through the coils. The dimensions of the coils used in different cases are mentioned in the respective figure captions.

\section{SELF PROTON PROBING OF NEUTRALISING CHARGE FLOW}

In a typical proton radiography technique, ${ }^{12,14}$ an intense laser pulse is focussed on a thin foil target to produce TNSA beam of protons from its rear surface. The quasilaminar, divergent beam of protons produced from the point-

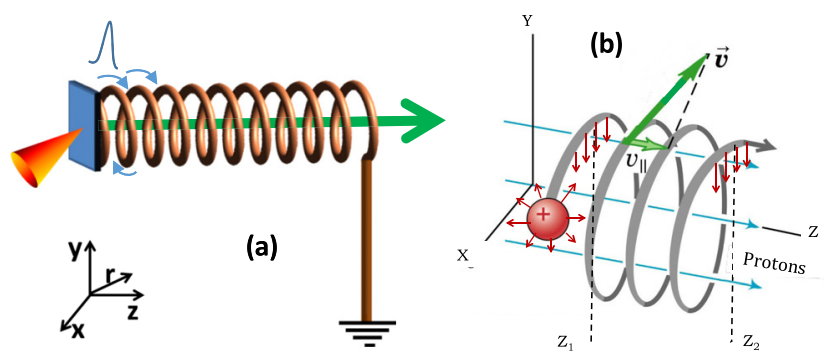

FIG. 2. (a) Schematic showing the helical coil target used for control and optimisation of the transiting proton beams. (b) Schematic showing the charge propagation with a velocity $\vec{v}$ along the windings of the helical coil, which give rise to a velocity $\overrightarrow{v_{\|}}$of the charge pulse along the axis of the coil depending on the coil diameter and pitch. The red arrows show the inwardly acting electric field on the transiting protons. 
like source is an ideal particle probe for point-projection imaging, with high spatial resolution, ${ }^{12}$ of an object (or plasma) placed within its field of view. Furthermore, the broad energy spectrum of the TNSA beam, coupled to its ultra-short burst duration (of the order of laser pulse duration) at the source, provides the unique capability of obtaining time resolved snapshots of the probed object/event. Protons with different energies cross the probed region at different times according to their time of flight. Due to the Bragg's peak energy deposition profile of protons inside material, a stack of RCFs placed at the detection plane will provide on different layers radiographs produced by different energy protons. The probing time at the center of the field of view for each RCF layer is mentioned at the top of each radiograph shown in Fig. 1(d)-1(k). The temporal resolution of the radiographs depends on several factors, such as the energy resolution of the RCF layer, the distance between the source and the object, and the width of the probed region causing proton deflection. For a typical setup, similar to the one discussed in this paper, the temporal resolution is of a few ps for $\mathrm{MeV}$ probe protons. ${ }^{12}$

The transient charging and discharging of laser irradiated targets have been studied previously using the standard proton probing technique (using two laser pulses), as reported in several papers. ${ }^{11,12,17}$ However, these previous investigations were limited to times close to the interaction and regions close to the laser interaction point. In particular, the initial stage of the propagation of an EM surface wave along the target surface was investigated and reported in Ref. 17. Here, we followed the propagation of the EM pulse at distances of several $\mathrm{cm}$ away from the interaction point and, by employing the SWP approach, over a cm length. Figs. 1(d)-1(k) show the data obtained in eight consecutive RCF layers within a stack detector. The darkness in the RCF images is proportional to the incident flux of protons arriving at the RCF. For an electrically neutral metal wire, the width of the shadow on the RCF will be approximately equal to the product of the diameter of the probed wire and the geometrical magnification $(M=L / l$, where $l$ and $L$ represent the distance from the proton source to the probed wire and the $\mathrm{RCF}$, respectively). In the case when the wire is electrically charged to a positive potential, the probe protons suffer strong Coulomb deflection, and the width of the proton depleted region (having lighter colors) on the RCF will depend on the strength of the electric field around the wire, i.e., on the local charge density of the wire, as well as the energy of the probe protons.

As can be seen in Figs. 1(d) and 1(e), the segments S4 and S5 appear to be charged to some positive potential, while the next wire segment, S6, remains electrically neutral. In the following time frames shown in Figs. 1(f) and 1(g), the segment S6 is becoming positively charged as evident from the increase of the width of the proton depleted region, compared to that observed in the earlier snapshots. This suggests that the EM pulse has flown from S5 to S6 during the time elapsed between the snapshots shown in Figs. 1(d) and $1(\mathrm{~g})$. At the same time, the segment S4 appears to gradually become electrically neutral from its left to the right hand side. At later probing times (as shown in Figs. 1(h)-1(k)), as expected, the pulse appears to have entered into the line segments S7, while the segments S4 and S5 are becoming completely neutral. Therefore, it is evident that the EM pulse has a finite temporal width, propagating away from the laser irradiated target with a high speed.

Quantitative information about the pulse temporal profile could be obtained from the data shown in Fig. 1. Following the procedure described in Ref. 13, the profile of the charge pulse along the wire was reconstructed by analysing the proton deflection at different points along the wire segments at different probing times. The charge pulse profile was measured to be of $\sim 7$ ps rise and $\sim 15$ ps decay (both half width at half maximum), with a peak linear charge density $\sim 12 \mu \mathrm{C} / \mathrm{m}$. The temporal profile of the charge pulse is consistent with the previous measurements of chargingdischarging of laser irradiated targets. ${ }^{12,17}$ The propagation speed of the peak was found to be $v_{\text {charge }}=(0.96 \pm 0.04) \mathrm{c}$, which agrees well with the speed of the charge front travelling away from the interaction point reported in Ref. 17.

\section{PROTON BEAM FOCUSSING BY HELICAL COIL LENS}

A modest reduction of proton beam divergence by exploiting the auto-charging of laser irradiated targets was demonstrated in Ref. 10, where the large surface area of the mm-scale guiding structures used significantly compromised the strength of the quasi-static focusing field inside the geometry, even for petawatt-laser interactions. Here, we show how an effective proton beam collimation can be achieved using the 10 TW TARANIS laser by exploiting the high amplitude EM pulse described in Sec. III.

This was achieved by creating a helical coil structure around the proton beam axis, as shown in Fig. 2(a). One side of the helical coil was directly connected to the irradiated foil with the other side connected to ground. Therefore, the EM pulse propagates through the windings of the helical coil, which provides a unidirectional flow of the neutralising current to the ground. Due to its finite duration, the pulse occupies only a few windings of the coil (depending on its diameter) at a given time, creating a highly localised region of strong electric field within the coil. The use of thin wires, compared to the mm-guiding structures used in Ref. 10, increases the localised charge density significantly and, hence, the strength of the electric field. Another promising feature of the helical coil lens is the ability to synchronise the flow of the charge pulse with the transiting protons. Depending on the radius $(R)$ and pitch $(d)$ of the coil, the electric field region will travel along the coil axis with a velocity $v_{\|}=(d / 2 \pi R) v_{\text {charge }}$, as shown in Fig. 2(b), that can be tuned to the velocity of transiting protons of a given energy. This enables effective beam guiding for a selected range of proton energies over an extended length of the coil (as the charge pulse was seen in Fig. 1 to have significant amplitude even after several $\mathrm{cm}$ of propagation).

Here, we present experimental results obtained from coils of different dimensions in order to elucidate the working principle of the helical coil lens. Fig. 3 highlights the salient features observed in the data. Where Fig. 3(b) shows the typical proton beam obtained from a flat foil target, the use of a helical 


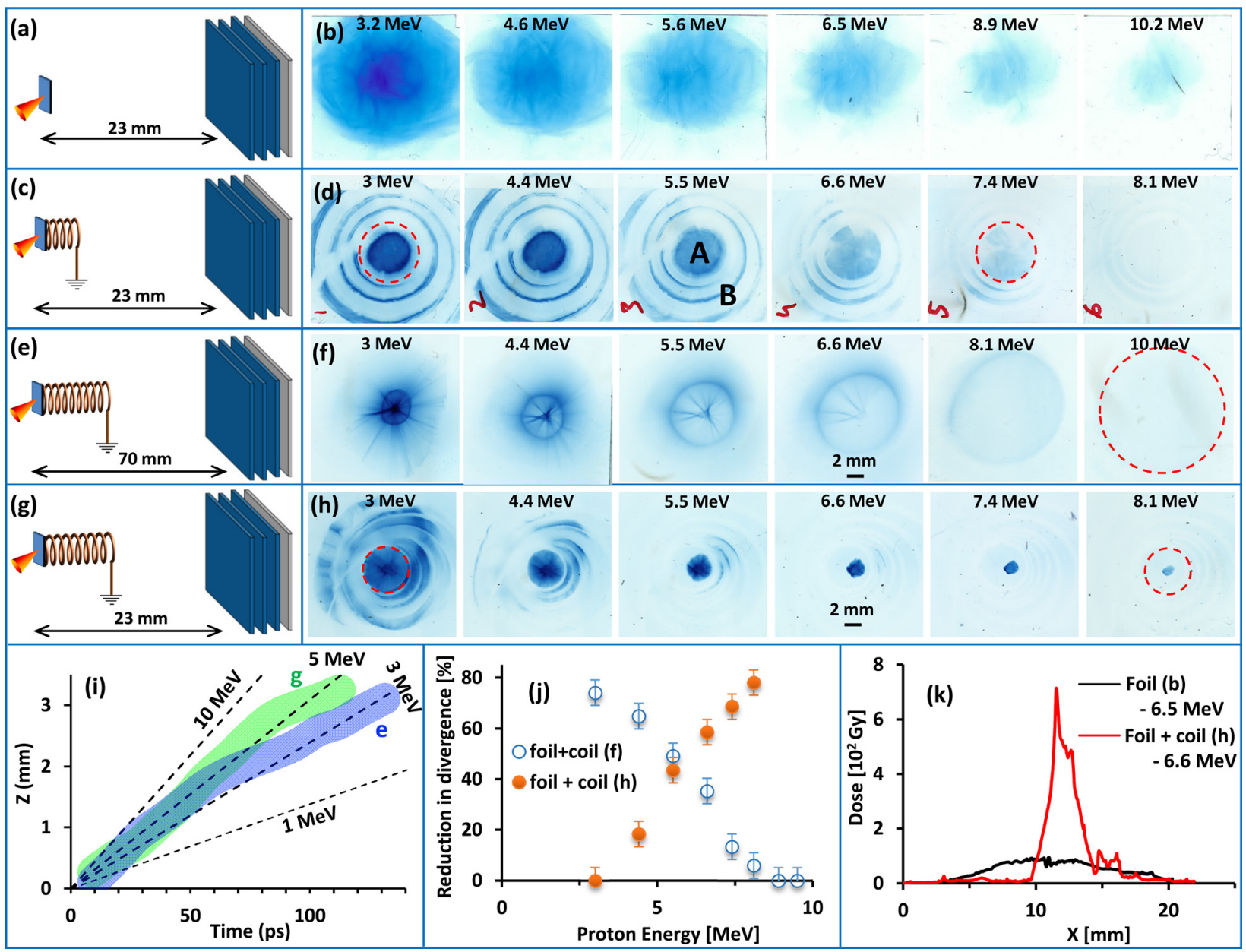

FIG. 3. (a), (c), (e), and (g) show the schematics for the four shots discussed in the text, for which the proton beam profile obtained in different RCF layers is shown in (b), (d), (f), and (h), respectively. The distance between the flat foil and the RCF stack was $\sim 70 \mathrm{~mm}$ for the case (e), instead of $\sim 23 \mathrm{~mm}$ in all other cases. The helical coils were made manually by winding $60 \mu \mathrm{m}$ aluminium wire over a straight wire of $900 \mu \mathrm{m}$ diameter. (i) Longitudinal (along the proton beam axis) position-time graphs for the peak of the pulse travelling along the coils in (e) and (g) (blue and green, respectively), by taking into account the measured pitch of the coils. The speed of the pulse along the coil windings was taken as 0.96 times the speed of light in vacuum, as estimated from the analysis of the data shown in Fig. 1. The lengths of the coils in case of (c), (e), and (g) were $\sim 1.8 \mathrm{~mm}, \sim 3.2 \mathrm{~mm}$, and $\sim 3.3 \mathrm{~mm}$, respectively. The thickness of the position-time curves represents the temporal bandwidth (7 ps rise and $15 \mathrm{ps}$ decay) of the pulse. The overlaid dashed lines show for a comparison the position vs. time of different energy protons (as labelled). The error in measuring, from the target images taken prior to the shots, the diameter, length, and pitch of the coils was $<50 \mu \mathrm{m}$. The red dashed circles in the RCF images in (d), (f), and (h) represent the geometrical projection of the exit winding of the respective coils on the RCF planes. The labels "A" and "B" in (d) point to the central part of the proton beam emerging through the coil and the shadows of coil windings produced by escaping protons through the coil windings. (j) Comparison between the reductions in beam divergence for different proton energies due to the differences in coil dimensions between the case (f) and (h). (k) Comparison between the dose profiles across the proton beam of similar energies obtained from the flat foil (b) and the foil + coil case (g)

coil at the rear side of the target (Figs. 3(c), 3(e) and 3(g)) significantly modifies the beam profile (Figs. 3(d), 3(f), and 3(h), respectively). For instance, the two pronounced features in the data shown in Fig. 3(d) are the central part of the beam (marked by "A") and the circular ring patterns around it (marked by "B"). Stronger deflection by the coil windings was observed for the lower energy protons due to the given diameter and pitch of this coil, which allowed synchronisation of the charge pulse with protons at the lower end of the spectrum. The central part of the proton beam (marked by " $\mathrm{A}$ ") is the part of the proton beam channelled through the helical coil. As expected, the diameter of the central part varied with the proton energy. Where the cone angle sustained by the central beam of $\sim 6 \mathrm{MeV}$ protons is similar to the cone angle sustained by the exit winding of the coil (marked by the red dashed circle in Fig. 3(d)), the diameter of the central beam for $3 \mathrm{MeV}$, for instance, indicates a significant reduction in proton beam divergence, of up to $\sim 30 \%$. By using a longer $(\sim 3.2 \mathrm{~mm}$ compared to $\sim 1.8 \mathrm{~mm}$ for the case in Fig. 3(c)) coil of similar diameter and pitch, the lower energy $(\sim 3 \mathrm{MeV})$ protons were strongly collimated producing a sub-mm spot size at $70 \mathrm{~mm}$ from the target rear surface, as shown in Fig. 3(f)). If one considers the size of the ring feature in the proton beam spatial profile, the lower energy protons undergo a reduction in beam divergence of $\sim 80 \%$, as shown in Fig. 3(j). By increasing the length of the helical coil, the focusing field was applied for a longer time to the synchronised bunch of protons and resulted in the observed beam collimation.

As mentioned earlier, the most promising feature of the helical coil lens is the ability to tune proton beam parameters by carefully choosing the dimensions of the helical coil. Fig. 3(f) shows a nearly collimated beam of high energy protons 
produced by the third coil, which was of similar length and diameter as the coil used in as Fig. 3(e). However, the pitch of the third coil was made slightly larger than the second one in order to synchronise with the protons towards the high energy end of the spectrum. The change in the synchronisation window (i.e., the energy range corresponding to proton velocities close to $v_{\|}$) by changing the pitch of the coil can be seen in Fig. 3(i). Although the pitches of the coils used in this experiment were not uniform across the coil length, the electric field region inside the coils shown in Figs. 3(e) and 3(g) was predominantly synchronised with 3 and $5 \mathrm{MeV}$ protons, respectively. The chromatic nature of the coil performance and its effective energy selection by changing its pitch is evident in Fig. 3(j), which compares the reduction in beam divergence vs proton energy between the two coils having different synchronisation windows. For the longer pitched coil, the reduction in beam divergence at $6.6 \mathrm{MeV}$ was $\sim 60 \%$, which led to a commensurate (nearly an order of magnitude) increase in particle flux compared to the flat foil target as shown in Fig. 3(h). For applicative purpose, the guided proton beam of narrow energy band exiting from the coil could be easily filtered out from the rest of the proton spectrum by using, for instance, a spatial aperture after the helical coil lens. Such a pencil beam of high flux, and possibly having a narrow energy bandwidth if one uses an optimised coil geometry, would be extremely useful for applications and further beam transport.

In order to understand the underlying working principle of the helical coil lens, the electric field distribution inside the helical coil was modelled numerically in cylindrical coordinate system. Using the same physical parameters for the coil as used in the experiment, a charge pulse of a given temporal profile was allowed to travel along it. The electric field at any given point at a given time was computed by adding the electric field vectors due to every small (for instance, one hundredth of its circumference) element of the coil, while considering the position of the charge pulse in the coil at the relevant probing time. Fig. 4 shows the electric field distribution inside the helical coil for the dimensions used in the case of Fig. 3(g), at three different times after the generation of the charge pulse (say, $t=0$ ). Due to the short duration of the charge pulse, the electric field distribution inside the coil is highly localised along the longitudinal axis of the coil. Assuming the broadband protons are also produced at $\mathrm{t}=0$ and travel along the coil axis, the time-of-flight dispersion of protons will allow energy selection by choosing the dimensions of the coil appropriately. As shown in Fig. 4 for the coil used in Fig. 3(g), protons around $5 \mathrm{MeV}$ were in sync with the charge pulse for the whole length of the coil, whereas the lower (higher) energy protons lag behind (lead) the charge pulse due to the time of flight dispersion.

There is another facet to the electric field produced by the travelling EM pulse in the coil. Due to the helical coil geometry, the electric field lines are curved inside the coil, which give rise to a longitudinal (along the coil axis) electric field component in addition to the transverse focusing field discussed above. The longitudinal field will act on the synchronised bunch of protons as they are being guided by the transverse focussing field, which, in principle, accelerate (decelerate) the leading (trailing) portion of the synchronised bunch. ${ }^{18}$ Since the charge pulse can travel along a wire over a long distance (significantly longer than that experimentally shown here), one can achieve a significant post-acceleration of the selected bunch of protons using a suitably long (cm, compared to the few $\mathrm{mm}$ coils used here) coil target. ${ }^{18}$

\section{SUMMARY AND DISCUSSIONS}

In summary, a scheme for simultaneous beam focussing, energy selection, and post-acceleration of laser driven proton beam is discussed. Following the prompt charge-up of a laser irradiated target, an ultrashort, high amplitude EM pulse flows through any components attached to the target. Experimental data show that the pulse can be transported over a long distance (several $\mathrm{cm}$ ) along a thin wire. The electric field associated with such pulse is strong enough to steer $\mathrm{MeV}$ protons and, hence, can be used to manipulate the laser driven $\mathrm{MeV}$ proton beams. Allowing the pulse to travel
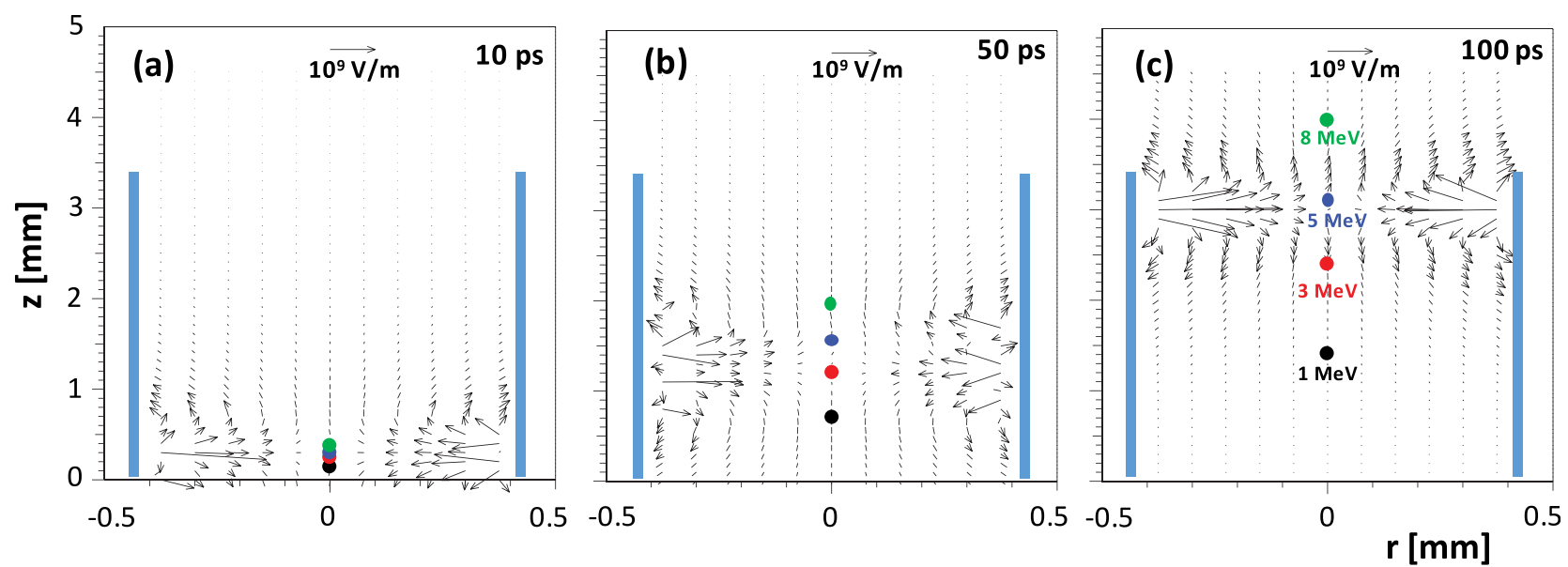

FIG. 4. (a)-(c) 2-dimensional map of electric field distribution across a longitudinal section of the coil at $10 \mathrm{ps,} 50 \mathrm{ps,}$ and $100 \mathrm{ps}$ after the laser interaction. The electric field at a given point is represented by an arrow of length proportional to the strength of the electric field. The arrow on the top of each image represents electric field of $10^{9} \mathrm{~V} / \mathrm{m}$. The blue solid lines at $r= \pm 0.43 \mathrm{~mm}$ represent the position of the coil. The black, red, blue, and green filled circles represent the positions of $1 \mathrm{MeV}, 3 \mathrm{MeV}, 5 \mathrm{MeV}$, and $8 \mathrm{MeV}$ protons, respectively, travelling along the axis of the coil. 
along a helical path around the proton beam, the transverse and longitudinal components of the electric field produced inside the helical coil can act simultaneously on a selected bunch (depending on the coil diameter and pitch) of the transiting protons to produce strong focussing and post-acceleration effects. ${ }^{18}$ The effect of the travelling EM pulse in a helical path towards chromatic focussing of laser driven protons is presented by varying the coil dimensions. Strong reduction in beam divergence (up to $75 \%$ reduction in divergence of $>5 \mathrm{MeV}$ protons) and a commensurate increase in beam flux were demonstrated using a few mm long coils of suitable diameter and pitch.

\section{ACKNOWLEDGMENTS}

The authors acknowledge funding from EPSRC [EP/ J002550/1-Career Acceleration Fellowship held by S.K., EP/ L002221/1, EP/K022415/1, EP/J500094/1, and EP/I029206/ 1], SBF-TR18 and GRK1203, EC-GA284464 and Invest Northern Ireland (POC-329). Authors acknowledge A. Schiavi (Univ. Roma 1, Italy) for the use of the particle tracing code, PTRACE. Data associated with research published in this paper can be accessible at http://dx.doi.org/ 10.17034/09ea3d74-f456-46e3-af9f-6e60cf0b07c0

${ }^{1}$ A. Macchi, M. Borghesi, and M. Passoni, Rev. Mod. Phys. 85, 751 (2013). ${ }^{2}$ F. Nürnberg, M. Schollmeier, E. Brambrink, A. Blažević, D. C. Carroll, K. Flippo, D. C. Gautier, M. Geißel, K. Harres, B. M. Hegelich, O. Lundh, K. Markey, P. McKenna, D. Neely, J. Schreiber, and M. Roth, Rev. Sci. Instrum. 80, 033301 (2009).

${ }^{3}$ S. V. Bulanov and T. Z. Esirkepov, Phys. Lett. A 299, 240 (2002); K. Ledingham and W. Galster, Br. J. Radiol. 80, 855 (2007); D. Doria, K. F. Kakolee, S. Kar, S. K. Litt, F. Fiorini, H. Ahmed, S. Green, J. C. G. Jeynes, J. Kavanagh, D. Kirby, K. J. Kirkby, C. L. Lewis, M. J. Merchant, G. Nersisyan, R. Prasad, K. M. Prise, G. Schettino, M. Zepf, and M. Borghesi, AIP Adv. 2, 011209 (2012).

${ }^{4}$ P. K. Patel, A. J. Mackinnon, M. H. Key, T. E. Cowan, M. E. Foord, M. Allen, D. F. Price, H. Ruhl, P. T. Springer, and R. Stephens, Phys. Rev. Lett. 91, 125004 (2003).

${ }^{5}$ S. Kar, A. Green, H. Ahmed, A. Alejo, A. P. L. Robinson, M. Cerchez, R. Clarke, D. Doria, S. Dorkings, J. Fernandez, S. R. Mirfyazi, P. McKenna, K. Naughton, D. Neely, P. Norreys, C. Peth, H. Powell, J. A. Ruiz, J. Swain, O. Willi, and M. Borghesi, New J. Phys. 18, 053002 (2016).

${ }^{6}$ T. Toncian, M. Borghesi, J. Fuchs, E. d'Humieŕes, P. Antici, P. Audebert, E. Brambrink, C. A. Cecchetti, A. Pipahl, L. Romagnani, and O. Willi, Science 312, 410 (2006).
${ }^{7}$ M. Schollmeier, S. Becker, M. Geißel, K. A. Flippo, A. Blažević, S. A. Gaillard, D. C. Gautier, F. Grüner, K. Harres, M. Kimmel, F. Nürnberg, P. Rambo, U. Schramm, J. Schreiber, J. Schütrumpf, J. Schwarz, N. A. Tahir, B. Atherton, D. Habs, B. M. Hegelich, and M. Roth, Phys. Rev. Lett. 101, 055004 (2008).

${ }^{8}$ S. Busold, D. Schumacher, O. Deppert, C. Brabetz, S. Frydrych, F. Kroll, M. Joost, H. Al-Omari, A. Blažević, B. Zielbauer, I. Hofmann, V. Bagnoud, T. E. Cowan, and M. Roth, Phys. Rev. Spec. Top. Accel. Beams 16, 101302 (2013).

${ }^{9}$ S. Kar, K. Markey, M. Borghesi, D. C. Carroll, P. McKenna, D. Neely, M. N. Quinn, and M. Zepf, Phys. Rev. Lett. 106, 225003 (2011).

${ }^{10}$ S. Kar, K. Markey, P. T. Simpson, C. Bellei, J. S. Green, S. R. Nagel, S. Kneip, D. C. Carroll, B. Dromey, L. Willingale, E. L. Clark, P. McKenna, Z. Najmudin, K. Krushelnick, P. Norreys, R. J. Clarke, D. Neely, M. Borghesi, and M. Zepf, Phys. Rev. Lett. 100, 105004 (2008).

${ }^{11}$ K. Quinn, P. A. Wilson, B. Ramakrishna, G. Sarri, L. Romagnani, A. Pipahl, O. Willi, L. Lancia, J. Fuchs, D. C. Carroll, M. N. Quinn, P. Gallegos, X. H. Yuan, P. McKenna, R. J. Clarke, D. Neely, M. Notley, A. Macchi, and M. Borghesi, Eur. Phys. J. D 55, 293 (2009).

${ }^{12}$ M. Borghesi, L. Romagnani, A. Schiavi, D. H. Campbell, M. G. Haines, O. Willi, A. J. MacKinnon, M. Galimberti, L. A. Gizzi, R. J. Clarke, and S. Hawkes, Appl. Phys. Lett. 82, 1529 (2003).

${ }^{13}$ H. Ahmed, S. Kar, G. Cantono, G. Nersisyan, S. Brauckmann, D. Doria, D. Gwynne, A. Macchi, K. Naughton, O. Willi, C. L. S. Lewis, and M. Borghesi, Nucl. Instrum. Methods A (published online).

${ }^{14}$ M. Borghesi, D. H. Campbell, A. Schiavi, M. G. Haines, O. Willi, A. J. MacKinnon, P. Patel, L. A. Gizzi, M. Galimberti, R. J. Clarke, F. Pegoraro, H. Ruhl, and S. Bulanov, Phys. Plasmas 9, 2214 (2002).

${ }^{15}$ T. Dzelzainis, G. Nersisyan, D. Riley, L. Romagnani, H. Ahmed, A. Bigongiari, M. Borghesi, D. Doria, B. Dromey, M. Makita, S. White, S. Kar, D. Marlow, B. Ramakrisha, G. Sarri, M. Zakaul-islam, M. Zepf, and C. L. S. Lewis, Laser Part. Beams 28, 451 (2010).

${ }^{16}$ D. Kirby, S. Green, F. Fiorini, D. Parker, L. Romagnani, D. Doria, S. Kar, C. Lewis, M. Borghesi, and H. Palmans, Laser Part. Beams 29, 231 (2011).

${ }^{17}$ K. Quinn, P. A. Wilson, C. A. Cecchetti, B. Ramakrishna, L. Romagnani, G. Sarri, L. Lancia, J. Fuchs, A. Pipahl, T. Toncian, O. Willi, R. J. Clarke, D. Neely, M. Notley, P. Gallegos, D. C. Carroll, M. N. Quinn, X. H. Yuan, P. McKenna, T. V. Liseykina, A. Macchi, and M. Borghesi, Phys. Rev. Lett. 102, 194801 (2009).

${ }^{18}$ S. Kar, H. Ahmed, R. Prasad, M. Cerchez, S. Brauckmann, B. Aurand, G. Cantono, P. Hadjisolomou, C. L. S. Lewis, A. Macchi, G. Nersisyan, A. P. L. Robinson, A. M. Schroer, M. Swantusch, M. Zepf, O. Willi, and M. Borghesi, Nat. Commun. 7, 10792 (2016).

${ }^{19}$ A. Alejo, H. Ahmed, A. Green, S. R. Mirfayzi, M. Borghesi, and S. Kar, Nuovo Cimento C 38, 188 (2015).

${ }^{20}$ L. Romagnani, M. Borghesi, C. A. Cecchetti, S. Kar, P. Antici, P. Audebert, S. Bandhoupadjay, F. Ceccherini, T. Cowan, J. Fuchs, M. Galimberti, L. A. Gizzi, T. Grismayer, R. Heathcote, R. Jung, TV. Liseykina, A. Macchi, P. Mora, D. Neely, M. Notley, J. Osterholtz, C. A. Pipahl, G. Pretzler, A. Schiavi, G. Schurtz, T. Toncian, P. A. Wilson, and O. Willi, Laser Particle Beams 26, 241 (2008). 